\title{
COMPACT LIE GROUP ACTION AND EQUIVARIANT BORDISM
}

\author{
SHABD SHARAN KHARE ${ }^{1}$
}

\begin{abstract}
Let $G$ be a compact Lie group and $H$ a compact Lie subgroup of $G$ contained in the center of $G$ with $H^{m}$ the maximal subgroup in the center, $H$ being $H$-boundary. Let $p_{r}: H^{m} \rightarrow H$ be the projection onto the $r$ th factor and $H_{r}$ be the $r$ th factor of $H^{m}$. Let $\left\{L_{r}\right\}$ be a family of subgroups of $G$ such that $L_{r} \cap H_{r}$ is nontrivial. Consider a $G$-manifold $M^{n}$ with $p_{r}\left(G_{x} \cap H^{m}\right)$ trivial or containing $L_{r}$, for every $x$ in $M^{n}$. The main result of the paper is that if $\forall x \in M^{n}, p_{r}\left(G_{x} \cap H^{m}\right)$ is trivial at least for one $r$, then $M^{n}$ is a $G$-boundary.
\end{abstract}

1. Introduction. This paper is a sequel to [3-5]. Conner and Floyd [1] proved that if $\mathbf{Z}_{2}^{k}$ acts on a closed manifold $M$ differentiably and without any fixed point, then $M$ is a boundary. Stong [ $\mathbf{7}]$ showed that if $(M, \theta)$ is a closed $\mathbf{Z}_{2}^{k}$-differential manifold without any stationary point, then $(M, \theta)$ is a $\mathbf{Z}_{2}^{k}$-boundary. In [3], we extended Stong's result for any finite abelian group of even order by proving the following. Let $G$ be a finite abelian group of even order, $(M, \theta)$ a closed $G$-differential manifold and the elementary 2-group $G_{2}$ in $G$ acts on $M$ under $\theta$ without any stationary point. Then $(M, \theta)$ is a $G$-boundary. In [4], we inititated this problem for nonabelian groups $S_{3}$ and dihedral groups. In [5], we have extended the result of [3] for an arbitrary finite group with center of even order. One needs the elementary 2-group $G_{2}(C)$ of the center of $G$ instead of $G_{2}$.

In the present note, we consider the action of a compact Lie group and prove that the induced action of the central elementary $H$-subgroup of $G$ determines $G$-bordism. This gives the results of $[\mathbf{3}-\mathbf{5}]$ in particular cases. ${ }^{1}$

I am thankful to Professor R. E. Stong for his invaluable suggestions. I am also thankful to the referee for very useful suggestions, which were instrumental in putting the paper in this form.

2. Preliminaries. Let $H$ be a compact Lie group. If there exists an $H$ differential closed manifold $(N, \theta)$ such that the boundary $\partial N=H$ and the restriction of the action $\theta$ to $H$ coincides with the operation in $H$, then we say that the compact Lie group $H$ is $H$-boundary. Let $G$ be a compact Lie group. By the central elementary $H$-group in $G$, we will mean the maximal subgroup $H^{n}(=H \times \cdots \times H$, $n$ times) contained in the center of $G$.

Consider a compact Lie group $G$ with $H^{n}$ the central elementary $H$-group in $G, H$ being $H$-boundary. Let us fix a point $h_{0}$ of $H$. Let $p_{r}: H^{n} \rightarrow H$ denote the projection onto the $r$ th factor, $1 \leq r \leq n$. Let $H_{r}$ denote the subgroup of $H^{n}$

Received by the editors August 2, 1982 and, in revised form, October 13, 1983.

1980 Mathematics Subject Classification. Primary 57R85.

Key words and phrases. $H$-boundary, admissible pair, $\left\{L_{r}\right\}$-type action, pseudo stationary point, equivariant bordism.

${ }^{1}$ The author was partially supported by DAE Grant. 
with $p_{i}\left(H_{r}\right)=H$, if $i=r$ and $p_{i}\left(H_{r}\right)=h_{0}$, if $i \neq r$. Consider a family $\left\{L_{r}\right\}$ of subgroups $L_{r}$ of $G$ such that $L_{r} \cap H_{r}$ is a nontrivial subgroup of $H_{r}, 1 \leq r \leq n$. By an $\left\{L_{r}\right\}$-type action of $G$, we mean a differential action of $G$ on a differential manifold such that for every $x$ in $M, p_{r}\left(G_{x} \cap H^{n}\right)$ is either trivial or contains $L_{r} \forall r$, $G_{x}$ being the isotropy group of $x$. A point $x$ in $M$ is said to be a pseudo stationary point if $p_{r}\left(G_{x} \cap H^{n}\right)$ is nontrivial $\forall r, 1 \leq r \leq n$.

A family $\mathcal{F}$ in $G$ is a collection of subgroups of $G$ such that if $K \in \mathcal{F}$, then all the subgroups of $K$ and all conjugates of $K$ are in $₹$. Let $\mathcal{F}^{\prime} \subset \mathcal{F}$ be families in $G$ such that there exists an $H$-boundary subgroup $H$ of $G$ satisfying the following conditions:

(a) no nontrivial subgroup of $H$ is contained in $K \forall K \in \mathcal{F}-\mathcal{F}^{\prime}$,

(b) the intersection $I$ of all the members of $\mathcal{F}-\mathcal{F}^{\prime}$ is in $\mathcal{F}-\mathcal{F}^{\prime}$,

(c) $H$ is contained in the center.

We call such a pair $\left(\mathcal{F}, \mathcal{F}^{\prime}\right)$ of families an admissible pair in $G$ with respect to $H \subset G$.

EXAMPLE 2.1. Consider a family $\left\{L_{r}\right\}$ of subgroups of $G$ such that $L_{r} \cap H_{r}$ is a nontrivial subgroup of $H_{r}$. Let $\mathcal{F}_{r}^{L}$ denote the family of all subgroups $K$ of $G$ for which $p_{j}\left(K \cap H^{n}\right)$ is trivial at least for one $j=1, \ldots, r$ and the nontrivial subgroups $p_{j}\left(K \cap H^{n}\right)$ of $H_{j}$ contain $L_{j}$. Then $\left(\mathcal{F}_{r+1}^{L}, \mathcal{F}_{r}^{L}\right)$ is an admissible pair of families in $G$ with respect to $H_{r+1}, 0 \leq r \leq n, \mathcal{F}_{0}^{L}$ being the empty family.

3. $\mathcal{F}_{n}^{L}$-free action and $G$-bordism. The object of this section is to show that if $(M, \theta)$ is an $\mathcal{F}_{n}^{L}$-free closed $G$-manifold, then $(M, \theta)$ is $G$-boundary. Let $\mathfrak{N}_{*}\left(G ; \mathcal{F}, \mathcal{F}^{\prime}\right)$ denote the $\left(\mathcal{F}, \mathcal{F}^{\prime}\right)$-free $G$-bordism group for a pair $\left(\mathcal{F}, \mathcal{F}^{\prime}\right)$ of families in $G$. For a given family $\mathcal{F}$ in $G$ and a subgroup $K$ of $G$, let $\mathcal{F}_{K}$ denote the smallest family in $G$ containing all the subgroups $[S \cup P], S \in \mathcal{F}$ and $P$ a subgroup of $K$.

THEOREM 3.1. If $\left(\mathcal{F}, \mathcal{F}^{\prime}\right)$ is an admissible pair of families in $G$ with respect to a subgroup $H$, which is $H$-boundary, then the homomorphism

$$
\mathfrak{N}_{*}\left(G ; \mathcal{\mp}, \mathcal{F}^{\prime}\right) \rightarrow \mathfrak{N}_{*}\left(G ; \mathcal{F}_{H}, \mathcal{F}_{H}^{\prime}\right)
$$

induced by the inclusion map $\left(\mathcal{F}, \mathcal{F}^{\prime}\right) \rightarrow\left(\mathcal{F}_{H}, \mathcal{F}_{H}^{\prime}\right)$ is the zero homomorphism.

Proof. Let $[M, \theta]$ be in $\mathfrak{N}_{n}\left(G ; \mathcal{F}, \mathcal{F}^{\prime}\right)$. Let $F$ denote the fixed points set of $I$ in $M, I$ being the intersection of all the members of $\mathcal{F}-\mathcal{F}^{\prime}$. Since $\mathcal{F}-\mathcal{F}^{\prime}$ is invariant under conjugation, $I$ is normal in $G$ so that the action $\theta$ induces an action on $F$, which we once again denote by $\theta$. Let $\nu$ be the normal bundle of the imbedding of $F$ in the interior of $M$ and $D(\nu)$ be the disc bundle with the action $\theta^{*}$ of $G$ on $D(\nu)$ induced by the real vector bundle maps covering the action $\theta$ on $F$. Since $F$ is the fixed points set of $I$, no nontrivial subgroup of $H$ is contained in $K \forall K \in \mathcal{F}-\mathcal{F}^{\prime}$, no point of $F$ will be fixed by the subgroup $[I \cup P], P$ being a nontrivial subgroup of $H$, so that $H$ will act freely on $F$ and hence on $D(\nu)$. Let $F^{\prime}=F / H$ and $D^{\prime}(\nu)=D(\nu) / H$. The actions $\theta$ and $\theta^{*}$ on $F$ and on $D(\nu)$ induce actions $\theta^{\prime}$ and $\theta^{* \prime}$ on $F^{\prime}$ and $D^{\prime}(\nu)$ respectively, because $H$ is contained in the center. Since $H$ acts freely on $F$ and $D(\nu)$, the quotient maps $\xi_{1}: F \rightarrow F^{\prime}$ and $\xi_{2}: D(\nu) \rightarrow D^{\prime}(\nu)$ are principal $H$-bundles. Since $H$ is $H$-boundary, there exists an $H$-differential closed manifold $(N, \emptyset)$ such that the boundary $\partial N=H$ and the restriction of the action $\emptyset$ to $H$ coincides with the operation in $H$. Consider the fibre bundles $\tilde{\xi}_{1}=\xi_{1}[N]$ and $\tilde{\xi}_{2}=\xi_{2}[N]$ associated to the principal $H$-bundles 
$\xi_{1}$ and $\xi_{2}$ respectively. The total space $E_{1}$ of $\tilde{\xi}_{1}$ is given by $E_{1}=(F \times N) / H$, where the action of $H$ on $F \times N$ is given by $h(m, t)=\left(m h, h^{-1} t\right), h \in H$ and $(m, t) \in F \times N$. Also the boundary $\partial E_{1}$ is diffeomorphic to $(F \times H) / H$. Let us take a fixed point $\tilde{h}$ of $H$. Define a map $\eta:(F \times H) / H \rightarrow F$ as $\eta([m, h])=m \bar{h}$, where $h=\bar{h} \tilde{h}$. Clearly $\eta$ is a diffeomorphism. Let us define an action $\psi_{1}$ of $G$ on $E_{1}$ as $g[m, t]=[m g, t]$. Then the diffeomorphism $\eta$ preserves the $H$-action. Thus $E_{1}$ is a $G$-manifold with $\partial E_{1}$ being equivariantly diffeomorphic to $F$. Similarly the total space $E_{2}$ of $\tilde{\xi}_{2}$ is $(D(\nu) \times N) / H$, where the action of $H$ on $D(\nu) \times N$ is given by $h(m, t)=\left(m h, h^{-1} t\right)$. Consider the action $\psi_{2}$ of $G$ on $E_{2}$ as $g[m, t]=[m g, t]$. Let $\alpha: E_{2} \rightarrow E_{1}$ be the map induced from $\nu^{\prime}: D^{\prime}(\nu) \rightarrow F^{\prime}$ by going to the fibre bundles; one has the commutative diagram:

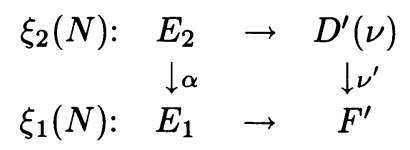

Also $\alpha^{-1}\left(\partial E_{1}\right)$ is diffeomorphic to $D(\nu)$ and the action $\psi_{2}$ on $\alpha^{-1}\left(\partial E_{1}\right)$ is isomorphic to the action $\theta^{*}$ on $D(\nu)$. Consider

$$
W=(M \times[0,1]) \cup E_{2} / \sim
$$

where $\sim$ is the equivalence relation in $W$ obtained by identifying $D(\nu) \times\{1\}$ with $\alpha^{-1}\left(\partial E_{1}\right)$. Let the action $\Phi$ of $G$ on $W$ be defined by $\Phi \mid M \times[0,1]=\theta \times 1$ and $\Phi \mid E_{2}=\psi_{2}$. Take $V$ to be

$$
(\partial M \times[0,1]) \cup\left(M \times\{1\}-(D(\nu) \times\{1\})^{\circ}\right) \cup\left(\partial E_{2}-\left(\alpha^{-1}\left(\partial E_{1}\right)\right)^{\circ}\right),
$$

where ${ }^{\circ}$ denotes the interior operator. Since $I$ is the intersection of all members of $\mathcal{F}-\mathcal{F}^{\prime}, V$ will be $\left(\mathcal{F}_{H}^{\prime}, \mathcal{F}_{H}^{\prime}\right)$-free. Also $W$ is $\left(\mathcal{F}_{H}, \mathcal{F}_{H}^{\prime}\right)$-free and $\partial W$ is diffeomorphic to $M \cup V$ identifying $\partial V$ with $\partial M$. This shows that $[M, \theta]$ is zero in $\mathfrak{N}_{*}\left(G ; \mathcal{F}_{H}, \mathcal{F}_{H}^{\prime}\right)$.

Let $\mathfrak{A}$ denote the family of all subgroups of $G$. Following the notations of Example 2.1 and using Theorem 3.1 we get the following

COROllaRY 3.2. For every $r, 0 \leq r \leq n$, the homomorphism $\mathfrak{N}_{*}\left(G ; \mathcal{F}_{r+1}^{L}, \mathcal{F}_{r}^{L}\right)$ $\rightarrow \mathfrak{N}_{*}\left(G ; \mathfrak{A}, \mathcal{F}_{r}^{L}\right)$ induced from the inclusion map $\left(\mathcal{F}_{r+1}^{L}, \mathcal{F}_{r}^{L}\right) \rightarrow\left(\mathfrak{A}, \mathcal{F}_{r}^{L}\right)$ is the zero one.

ProOF. Since $\left(\mathcal{F}_{r+1}^{L}, \mathcal{F}_{r}^{L}\right)$ is an admissible pair of families with respect to the subgroup $H_{r+1}, 0 \leq r<n$, and $\left(\mathcal{F}_{r}^{L}\right)_{H_{r+1}}=\mathcal{F}_{r}^{L}$, Theorem 3.1 gives the corollary.

COROLlaRY 3.3. Let $M$ be a closed $G$-manifold with $\left\{L_{r}\right\}$-type of action for some family $\left\{L_{r}\right\}$ of subgroups of $G$ such that $L_{r} \cap H_{r}$ is nontrivial. If $M$ does not have any pseudo stationary point, then $M$ is a $G$-boundary.

PrOOF. It is enough to show that the homomorphism $\mathfrak{N}_{*}\left(G ; \mathcal{F}_{n}^{L}\right) \rightarrow \mathfrak{N}_{*}(G ; \mathfrak{A})$ induced from the inclusion map $\mathcal{F}_{n}^{L} \rightarrow \mathfrak{A}$ is the zero one. By Corollary 3.2 and the exact bordism sequence for the triple $\left(\mathfrak{A}, \mathcal{F}_{r+1}^{L}, \mathcal{F}_{r}^{L}\right)$, one gets that $j_{*}: \mathfrak{N}\left(G ; \mathfrak{A}, \mathcal{F}_{r}^{L}\right)-$ $\mathfrak{N}_{*}\left(G ; \mathfrak{A}, \mathcal{F}_{r+1}^{L}\right)$ is a monomorphism, $j:\left(\mathfrak{A}, \mathcal{F}_{r}^{L}\right) \rightarrow\left(\mathfrak{A}, \mathcal{F}_{r+1}^{L}\right)$ is the inclusion map. Therefore the composite

$$
\mathfrak{N}_{*}\left(G ; \mathfrak{A}, \mathcal{F}_{0}^{L}\right) \rightarrow \mathfrak{N}_{*}\left(G ; \mathfrak{A}, \mathcal{F}_{1}^{L}\right) \rightarrow \cdots \rightarrow \mathfrak{N}_{*}\left(G ; \mathfrak{A}, \mathcal{F}_{n}^{L}\right)
$$


is a monomorphism and hence by the bordism exact sequence of the triple $\left(\mathfrak{A}, \mathcal{F}_{n}^{L}, \mathcal{F}_{0}^{L}\right)$, one gets that $\mathfrak{N}_{*}\left(G ; \mathcal{F}_{n}^{L}, \mathcal{F}_{0}^{L}\right) \rightarrow \mathfrak{N}_{*}\left(G ; \mathfrak{A}, \mathcal{F}_{0}^{L}\right)$ is the zero homomorphism. This completes the proof, since $\mathcal{F}_{0}^{L}$ is empty.

REMARK 3.4. Taking $G$ to be a finite abelian group of even order and $H$ to be $\mathbf{Z}_{2}$, one gets Corollary 3.7 of [3]. Considering $G$ to be a finite group with center of even order and $H$ to be $\mathbf{Z}_{2}$, one gets Corollary 3.3 of [5].

REMARK 3.5. The case $H=\mathbf{Z}_{2}$ can also be obtained in a simpler way using the technique used in the Appendix of [2]. The case $G=$ finite group and $H=\mathbf{Z}_{2}$, has been obtained by Kosniowski [6] using the concept of slices.

\section{REFERENCES}

1. P. E. Conner and E. E. Floyd, Differentiable periodic maps, Springer-Verlag, Berlin, 1964.

2. G. C. Hamrick and D. C. Royster, Flat Riemannian manifolds are boundaries, Invent. Math 66 (1982).

3. S. S. Khare, $\left(\mathcal{F}, \mathcal{F}^{\prime}\right)$-free bordism and stationary points set, Internat. J. Math. Math. Sci. (to appear).

4. __ Stationary points set and G-bordism, Indian J. Pure Appl. Math. 14 (1983), 1-4.

5. __ Finite group action and equivariant bordism Pacific J. Math. (to appear).

6. C. Kosniowski, Some equivariant bordism theories vanish, Math. Ann. 242 (1979).

7. R. E. Stong, Equivariant bordism and $\left(\mathbf{Z}_{2}\right)^{k}$-actions, Duke Math. J. 37 (1970).

Department of Mathematics, North-Eastern Hill University, Bijni Campus, Bhagyakul ROAD, LAITUmKhrah, SHILlONg 793 003, MEghalaya India 